\title{
Electromigration Lifetimes and Void Growth at low Cumulative Failure Probability
}

\author{
Hideaki Tsuchiya and Shinji Yokogawa \\ Test Analysis Technology Development Division, NEC Electronics Corporation, 1753 Shimonumabe, Nakahara, \\ Kawasaki, Kanagawa 211-8668, Japan \\ Fax:+81-44-435-1873,E-mail: hideaki.tsuchiya@necel.com
}

\begin{abstract}
We studied electromigration (EM) lifetimes and void growth at low cumulative failure probability. We carried out EM test in damascene $\mathrm{Cu}$ lines using sudden-death type test structures. Its cumulative failure probability ranges from 0.005 to $90 \%$. To investigate the void growth behaviour, $\mathrm{Cu}$ microstructures was investigated. EM lifetime shows correlation with the void nucleation site and the void volume. In addition, the nucleation site is affected by the characteristics of grain boundary near vias.
\end{abstract}

\section{Introduction}

Electromigration (EM) remains a major reliability concern due to the aggressive scaling of interconnects and increasing numbers of vias. So, it is important to understand EM lifetime distribution and void growth behaviour at low cumulative failure.

In this study, we tested the damascene $\mathrm{Cu}$ interconnects using sudden-death type test structures [1,2]. Its cumulative failure probability ranges from 0.005 to $90 \%$. We discuss EM lifetime distribution and the void growth behaviour.

\section{Experiments}

We used three types of sudden-death test structures to investigate failures at low cumulative probability. They consist of multi-link line/via elements with 2, 20 and 2000 links. EM test was performed at $350^{\circ} \mathrm{C}$ with a current density of $1.7 \mathrm{MA} / \mathrm{cm}^{2}$.

\section{Results and Discussion}

Fig. 1 shows the cumulative failure probability plots of lifetime for each test structures. It shows Weibull distribution and lognormal distribution. Three types of test structures are plotted on the one distribution curve. This result suggests that each failure modes are the same. As compared to the Weibull curve, the lognormal curve fits for a straight line. In other words, the lifetimes are well represented by lognormal distribution in wide cumulative failure range.

Many previous works reported that $\mathrm{Cu} /$ capdielectric interface is a dominant diffusion path [3]. In addition, the cross-section of void indicates that void formation is sensitive to grain boundary. We investigated the characteristics of $\mathrm{Cu}$ grain with electron backscatter diffraction (EBSD) technique.

Fig.2 shows a cross-sectional scanning transmission electron microscopy (S-TEM) image and an orientation map of grains near a void. The void edge is straight in the vicinity of $\Sigma 3$ grain boundary. On the other hand, the edge is bended at 
the $51^{\circ}$ wide angle boundary. These results suggest that $\Sigma 3$ boundary does not make contribution to void growth. Wide angle boundaries seem to contribute to a void nucleation, conversely. It is consistent with the previous work about Al interconnects [4]. Wetzig et al. proposed the following; the dominant path of $\mathrm{Al}$ diffusion is wide angle grain boundary and $\Sigma 3$ boundaries do not act as paths with high material transport.

In Fig.3, EM lifetimes show positive correlation with the void volume. Furthermore, the results of cross-sectional analysis imply that the void volume depends on the nucleation site. In addition, the nucleation site depends on the site of wide angle grain boundary. Eventually, the wide angle grain boundary under vias is the worst case for EM lifetime.

\section{Conclusion}

We reported about EM lifetimes and void growth at low cumulative failure probability. Observed EM lifetimes show strong correlation with the void volumes. Wide angle grain boundary under vias behaves as a nucleation site at low cumulative failure probability.

\section{References}

[1] M. Gall, C. Capasso, D. Jawarni, R. Hernandez, H. Kawasaki, and P. S. Ho, J. of Appl. Phys., Vol. 90, pp. 732-740 (2001).

[2] S. Yokogawa, Journal of Reliability Engineering Association of Japan, Vol. 25, No. 8, pp. 811-820 (2003).

[3] S. Yokogawa, N. Okada, Y. Kakuhara, and H. Takizawa, Microelectronics Reliability, Vol. 41, pp. 1409-1416 (2001).

[4] K. Weitz, H. Wendrock, A. Buerke, and T. Kotter, Proc. of $5^{\text {th }}$ Int. Workshop on Stress-Induced Phenomena in Metallization, pp. 89-99 (1999).

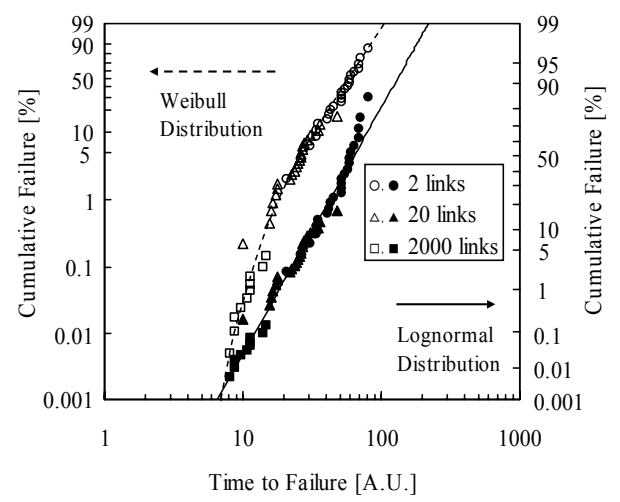

Fig. 1. Cumulative failure plots of lifetime. EM lifetimes are well represented by lognormal distribution.
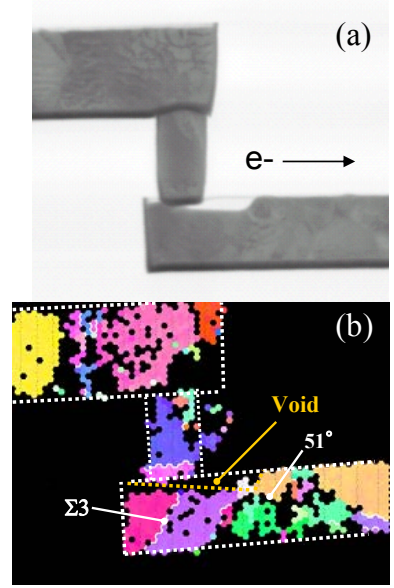

Fig. 2. Cross-sectional observation of the void at the cathode end of M1. (a) S-TEM image. (b) Orientation map including grain boundary classification.

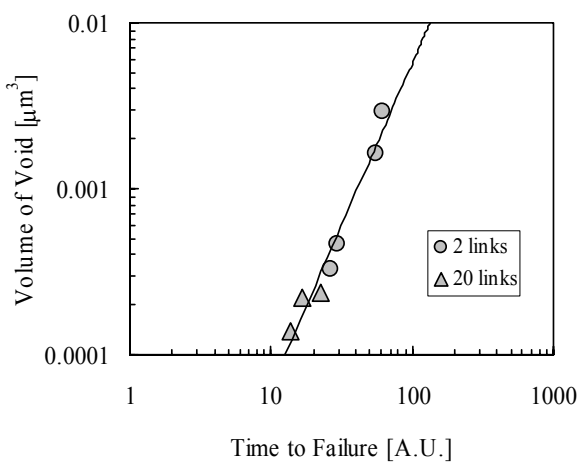

Fig. 3. The void volume show positive correlation with EM lifetimes. 